\title{
Article \\ Development of Multifunctional Myoelectric Hand Prosthesis System with Easy and Effective Mode Change Control Method Based on the Thumb Position and State
}

\author{
Sung-Yoon Jung ${ }^{1}$, Seung-Gi Kim ${ }^{2}$, Joo-Hyung Kim ${ }^{1}$ and Se-Hoon Park ${ }^{3, *} \mathbb{D}$ \\ 1 Department of Mechanical Engineering, Inha University, Incheon 22201, Korea; \\ syjung0410@gmail.com (S.-Y.J.); joohyung.kim@inha.ac.kr (J.-H.K.) \\ 2 Department of Electrical, Electronics \& Communication Engineering, Koreatech University, \\ Incheon 22201, Korea; hnlsg211@gmail.com \\ 3 Korea Orthopedics \& Rehabilitation Engineering Center, Incheon 21417, Korea \\ * Correspondence: mbb1122@naver.com; Tel.: +82-10-8776-7278; Fax: +82-032-512-9794
}

check for

updates

Citation: Jung, S.-Y.; Kim, S.-G.; Kim, J.-H.; Park, S.-H. Development of Multifunctional Myoelectric Hand Prosthesis System with Easy and Effective Mode Change Control Method Based on the Thumb Position and State. Appl. Sci. 2021, 11, 7295. https://doi.org/10.3390/app11167295

Academic Editor: Alessandro Gasparetto

Received: 29 June 2021

Accepted: 3 August 2021

Published: 9 August 2021

Publisher's Note: MDPI stays neutral with regard to jurisdictional claims in published maps and institutional affiliations.

Copyright: (c) 2021 by the authors. Licensee MDPI, Basel, Switzerland. This article is an open access article distributed under the terms and conditions of the Creative Commons Attribution (CC BY) license (https:// creativecommons.org/licenses/by/ $4.0 /)$.

\begin{abstract}
Commercial multi-degrees-of-freedom (multi-DOF) myoelectric hand prostheses can perform various hand gestures and grip motions using multiple DOFs. However, as most upper limb amputees have less than two electromyogram (EMG) signals generated at the amputation site, it is difficult to control various hand gestures and grip motions using multi-DOF myoelectric hand prostheses. This paper proposes a multifunctional myoelectric hand prosthesis system that uses only two EMG sensors while improving the convenience of upper limb amputees in everyday life. The proposed system comprises a six-DOF myoelectric hand prosthesis and an easy and effective control algorithm that enables upper limb amputees to perform various hand gestures and grip motions. More specifically, the hand prosthesis has a multi-DOF five-finger mechanism and a small controller that can be mounted inside the hand, allowing it to perform various hand gestures and grip motions. The control algorithm facilitates four grip motions and four gesture motions using the adduction and abduction positions of the thumb, the flexion and extension state of the thumb, and three EMG signals (co-contraction, flexion, and extension) generated using the two EMG sensors. Experimental results indicate that the proposed system is a versatile, flexible, and effective hand prosthesis system for upper limb amputees.
\end{abstract}

Keywords: myoelectric hand prosthesis system; multi-DOF myoelectric hand prosthesis; easy and effective control algorithm

\section{Introduction}

Humans use their hands not only to grip objects but also to communicate and express emotions through various hand motions [1]. Upper limb amputees lose such hand functions, and therefore, many of them feel uncomfortable in their daily life. Upper limb amputees are typically fitted with a prosthetic hand to compensate for their lost hand functions. Unfortunately, although a cosmetic prosthetic hand has a similar shape to a human hand, it cannot perform basic grip functions. Further, a body-powered prosthetic hand is difficult to use in daily life because larger body motions are required to perform a simple grip motion. In an effort to resolve such issues, Ottobock developed a myoelectric hand prosthesis called the MYO hand in 1987 [2]. The MYO hand can grasp objects easily and safely using the electromyogram (EMG) signals from residual muscles on an amputated upper limb, without requiring any additional equipment. However, it cannot execute the same range of motions as that executed by human hands because it has less than two degrees of freedom (DOFs), owing to the use of just one actuator. Recently, multiple-degrees-of-freedom (multi-DOF) myoelectric hand prostheses with four or more embedded actuators in the finger phalange or palm have been developed [3-7]. These hands can perform various hand gestures and grip motions in a manner that is similar 
to the human hand owing to their additional DOFs. However, because it is difficult to control multi-DOF myoelectric hand prostheses with the existing control method [8,9], many researchers utilize a pattern-recognition-based control method that can perform various hand movements using the EMG signals collected from the residual muscle at the amputation site [10-21]. The techniques employed incorporate various types of EMG pattern feature extractions and classifiers to achieve a classification accuracy of more than $95 \%$ for pattern recognition (PR) control of the multi-DOF myoelectric hand prosthesis [22]. However, PR control is difficult to use commercially owing to variabilities such as changes in arm posture, level of muscle contraction, and various amputation site shapes and lengths for upper limb amputees [23-25]. Moreover, most upper limb amputees have a limited number of EMG signals generated at the amputation site [10,26], making it difficult to perform various hand gestures and grip motions using the multi-DOF myoelectric hand prosthesis with PR control methods.

Consequently, the manufacturers of Bebionic hand [2] and i-LIMB hand [27] use mode switching control with just two EMG sensors to control the multi-DOF hand prosthesis. However, it is very difficult for users to master this control method because there is no association between the mode switching control command and the method of performing various hand motions. Furthermore, even when they can perform these hand motions, the time delay and method are unnatural. To solve these problems, the manufacturers have proposed different interfaces for controlling these hands, such as mobile phone applications and small coin-shaped chips attached to certain objects. However, these interfaces require the user to press a button on the phone application during actual conversations, thus disrupting the conversation flow. Therefore, these interfaces are inconvenient for use in everyday life.

To solve the problems outlined above, this paper proposes a multifunctional myoelectric hand prosthesis system that can perform eight hand gestures and grip motions using only two EMG sensors, without requiring the training of additional devices or other control methods for users. The proposed system consists of a hand prosthesis with five fingers, a controller equipped with a six-axis motor driver, and an easy and effective control algorithm that enables upper limb amputees to perform various hand gestures and grip motions using a multi-DOF myoelectric hand prosthesis. Most upper limb amputees have less than two EMG signals emerging from the amputation site. Therefore, the proposed easy and effective control algorithm allows the performing of eight hand gestures and grip motions according to the thumb adduction and abduction motion state and the thumb flexion and extension state using three control commands generated from only two EMG sensors. In this study, to verify the practicality of the algorithm, an upper limb amputee performed four hand gestures and four grip motions using a hand with six DOFs.

The remainder of this paper is organized as follows. Section 2 describes the developed multi-DOF myoelectric hand prosthesis system. Section 3 presents the easy and effective control algorithm for easily and efficiently using the hand. Section 4 describes experiments conducted using the algorithm. The experimental results show that the developed hand and algorithm enable versatile and flexible hand movement by amputees.

\section{Method}

\subsection{Multi-DOF Myoelectric Hand Prosthesis}

In this study, the fingers of the multi-DOF myoelectric hand prosthesis were designed based on the anatomical structure and motion mechanism of human fingers. Two types of fingers were designed, i.e., the thumb and the other four fingers. As shown in Figure 1a, the thumb consists of one phalange and two joints-namely, the metacarpophalangeal (MCP) joint and trapeziometacarpal (TM) joint. As shown in Figure 1b, the other four fingers consist of three phalanges (the distal phalange (DP), middle phalange (MP), and proximal phalange (PP)), one bone (the metacarpal bone (MB)), and two joints connecting the phalange and the bone (the proximal interphalangeal (PIP) and MCP joints). 


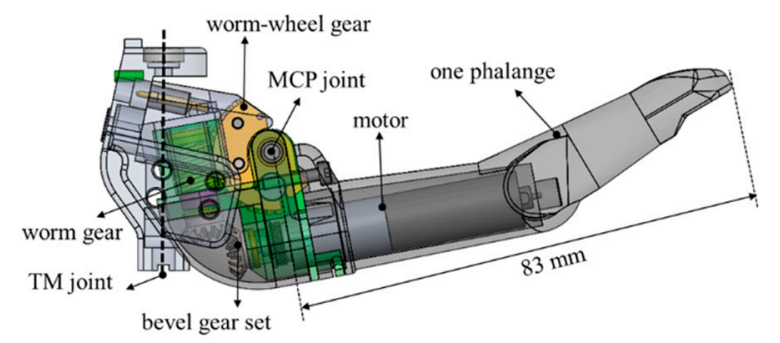

(a)

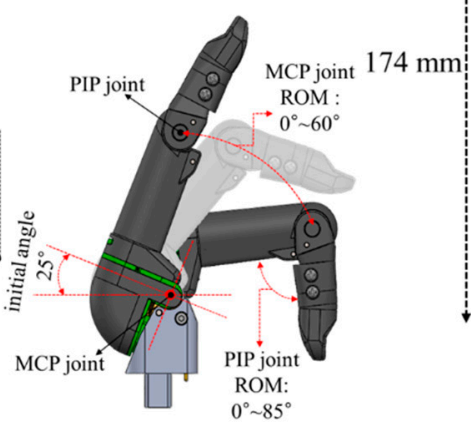

(b)

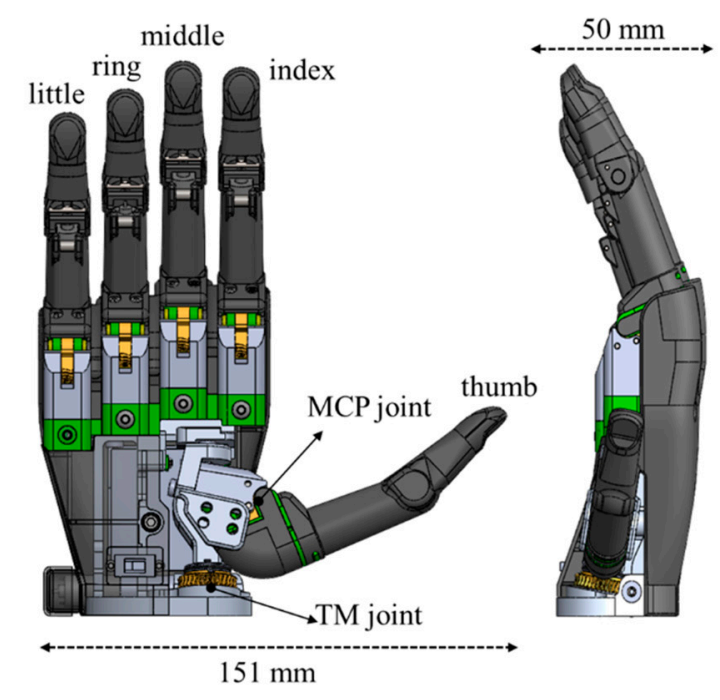

(c)

Figure 1. Structure of the proposed multi-DOF myoelectric hand prosthesis: 3D schematic of the (a) thumb, (b) other fingers, and (c) schematic of the multi-DOF myoelectric hand prosthesis.

The flexion and extension motions of the human finger are performed by pulling the tendon fixed to the phalange as the muscle contracts. In this study, we use a motor as a muscle and a wire as a tendon to perform each finger flexion motion. The motor is mounted inside the PP of each finger, and the wires are fixed to the worm-wheel gear and the MP. The designed finger flexion motion is performed by pulling the wire affixed to the worm-wheel gear when the motor is driven. The PP of the finger is actuated to $60^{\circ}$ from the MCP joint by the motor-gear mechanism (see Figure $1 \mathrm{~b}$ ). At this time, the wire affixed to the worm-wheel gear is pulled such that the MP and DP synchronously actuate the PP by $85^{\circ}$. The extension motion of the finger is performed by a restoring spring mounted between the PP and the MP. The thumb can perform flexion, extension, and rotation motions. The flexion motion of the thumb is performed by the MCP joint using the motor-gear mechanism. Further, the rotation motion is performed by the TM joint using the motor-gear mechanism in the palm of the hand.

The human hand has 22 DOFs and weighs about $400 \mathrm{~g}$ [28]. The proposed hand was developed based on the human hand, as shown in Figure 1c. The developed hand was manufactured using AL 6061 and SUS 304 materials to reduce the total weight and increase the durability of the hand. Its dimensions are $151 \times 174 \times 50 \mathrm{~mm}(\mathrm{~W} \times \mathrm{L} \times \mathrm{H})$. The hand performs various hand gestures and grip motions using the five fingers with the gear-motor-wire mechanism. It has six DOFs; specifically, the thumb has two DOFs, and the other four fingers have one DOF each. Further, this hand, including the motor, weighs about $503 \mathrm{~g}$ (see Figure 2). The proposed hand has a faster flexion angular velocity than other commercial multi-DOF myoelectric hand prostheses, and its maximum gripping force is on par with that of other commercial products (see Table 1). 


\begin{tabular}{|c|c|c|}
\hline \multirow{9}{*}{ thumb } & $=$ & $\begin{array}{l}\text { Multi-DOF myoelectric hand } \\
\text { prosthesis }\end{array}$ \\
\hline & fingers & 5 \\
\hline & DOF & 6 \\
\hline & size & $\begin{array}{c}151 \times, 174 \times, 50 \mathrm{~mm} \\
(\mathrm{~W} \times, \mathrm{L} \times, \mathrm{T})\end{array}$ \\
\hline & flexion speed & $60^{\circ} / 0.58 \mathrm{~s}$ \\
\hline & normal grip force & $40 \mathrm{~N}$ or more \\
\hline & power grip force & $120 \mathrm{~N}$ or more \\
\hline & weight & $\begin{array}{c}503 \mathrm{~g} \\
\text { (including actuators) }\end{array}$ \\
\hline & battery & $7.4 \mathrm{~V}, 2000 \mathrm{mAh}$ \\
\hline
\end{tabular}

Figure 2. Photograph of the developed multi-DOF hand prosthesis.

Table 1. Comparison of specifications with commercial multi-DOF myoelectric hand prostheses.

\begin{tabular}{|c|c|c|c|c|c|}
\hline $\mathrm{C}^{\text {Specification }}$ & $\begin{array}{c}\text { Size } \\
(\mathrm{W} \times, \mathrm{L})(\mathrm{mm})\end{array}$ & $\begin{array}{l}\text { Power Grip } \\
\text { Force } \\
\text { (N) }\end{array}$ & $\begin{array}{l}\text { Precision Grip } \\
\text { Force } \\
\text { (N) }\end{array}$ & $\begin{array}{c}\text { Finger } \\
\text { Flexion/Extension } \\
\text { Angular Velocity } \\
(\% / \mathrm{s})\end{array}$ & $\begin{array}{l}\text { Weight } \\
\text { (g) }\end{array}$ \\
\hline $\begin{array}{c}\text { Developed } \\
\text { hand prosthesis } \\
\text { (Figure } 2)\end{array}$ & $73.6 \times, 174$ & 120 or more & 37.5 & $60 / 0.58$ & 503 \\
\hline i-LIMB hand [27] & $\begin{array}{c}74.5 \times, 182.5 \\
(\text { small size })\end{array}$ & 136 & 10.8 & $81.8 / 1$ & 504 \\
\hline Bebionic hand [2] & $\begin{array}{c}72 \times, 165 \\
\text { (medium size) }\end{array}$ & 140 & 34 & $96.4 / 1$ & 591 \\
\hline Vincent hand [3] & $75 \times, 160$ & & & $103.3 / 1$ & \\
\hline
\end{tabular}

\subsection{Controller}

In the developed multi-DOF myoelectric prosthetic hand, a motor is mounted inside the PP of all five fingers, and each finger performs flexion motion when the motor is driven. In this study, a controller with a six-axis motor driver was designed to independently perform the flexion motion of each finger. The designed controller consists of an EMG sensor interface for measuring EMG signals, a micro controller unit (MCU), motor driver, a current feedback sensor, a battery, and a Bluetooth communication module for the debugger (see Figure 3a). The EMG sensor interface is designed to detect two EMG signals from the residual muscles of the upper limb amputee, and the MCU was designed using a 32-bit CPU. The motor driver is designed with six axes to control the fingers independently, and the current feedback sensor is designed to measure the generated motor load when the finger is actuated. The battery voltage is $7.4 \mathrm{~V}$; the input voltages of the MCU and Bluetooth communication module are 5 and $3.3 \mathrm{~V}$, respectively, from the battery.

In this study, we developed a controller based on the block diagram for the multiDOF myoelectric prosthetic hand shown in Figure 3b. The developed controller uses the cortex-M4 micro-controller and can independently control six motors according to the EMG signals. In addition, the hand gestures and grip motions of the developed hand can be controlled through the feedback of motor load during finger actuation. The controller has dimensions of $38 \times 25 \mathrm{~mm}(\mathrm{~W} \times \mathrm{L})$. 


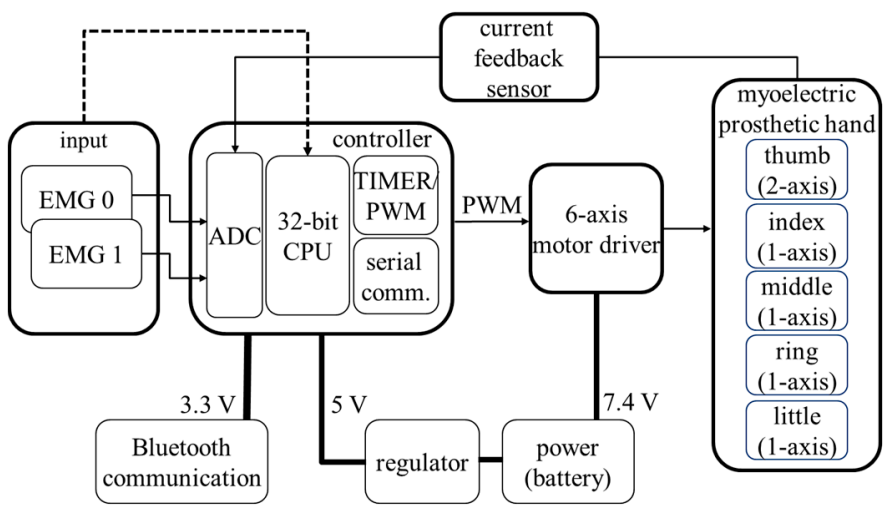

(a)

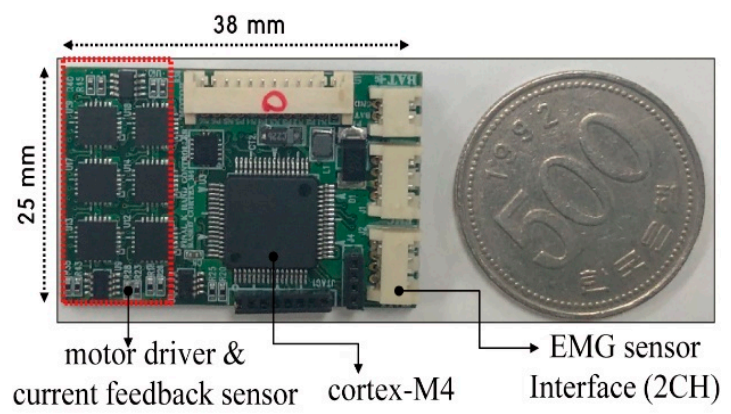

(b)

Figure 3. Developed controller with six-axis motor drivers: (a) controller block diagram and (b) developed controller.

\subsection{Flexion Speed and Grip Force of Developed Myoelectric Hand Prosthesis}

The flexion angular velocity of the finger and grip force of the hand were measured using the developed hand and controller. The flexion angular velocity of the finger is an important factor for performing hand gestures and grip motions. Therefore, the flexion angular velocity was measured by actuating the motor mounted inside the finger phalange. The motor used for the finger flexion motion was controlled by pulse width modulation (PWM). Consequently, the flexion angular velocity of the finger was $60 / 0.58 \mathrm{~s}$, which is presented in see Figure 4a.

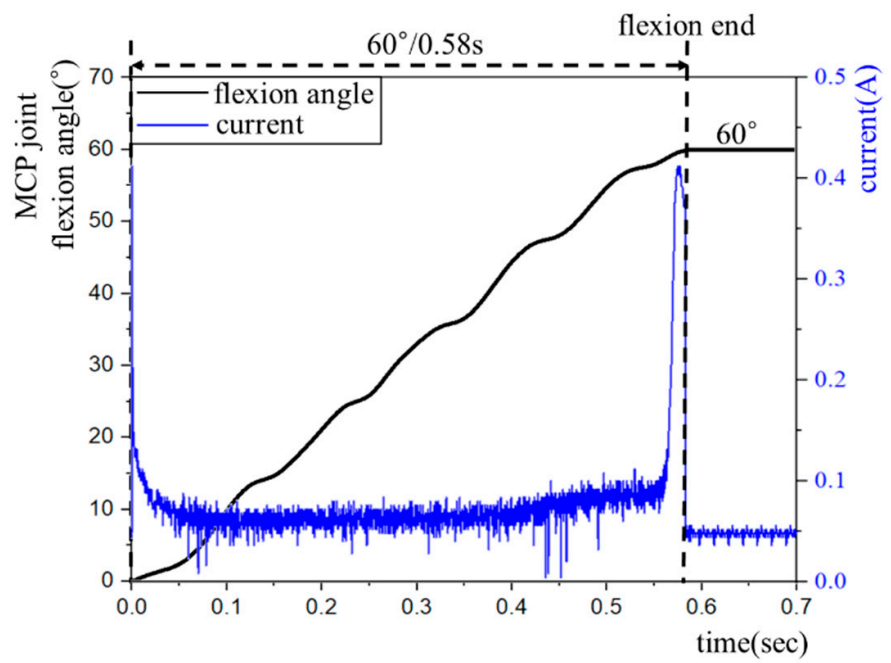

(a)

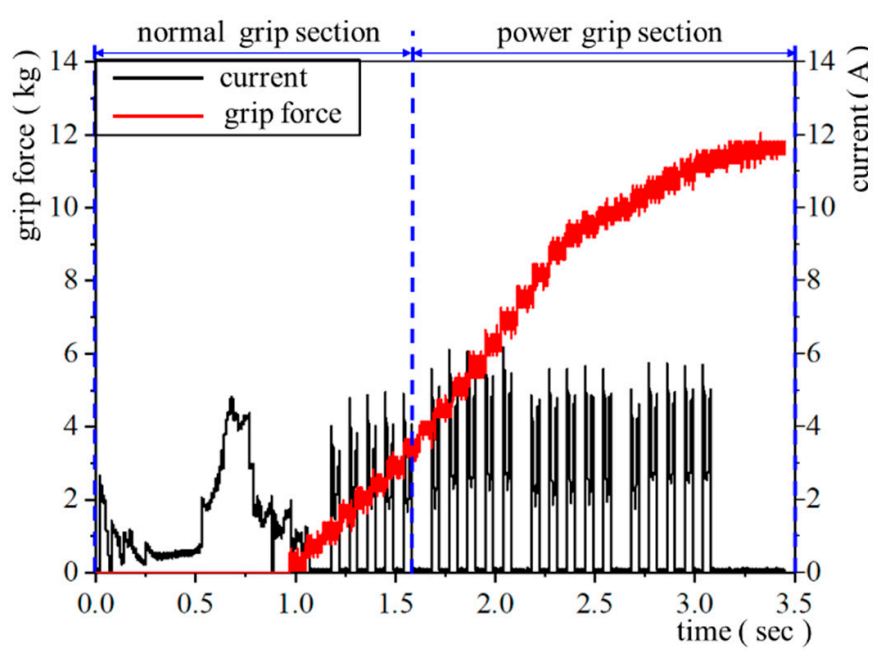

(b)

Figure 4. Flexion angular velocity and grip force of the developed myoelectric hand prosthesis: (a) ROM and flexion angular velocity of the MCP joint of the finger. The black line shows the MCP joint flexion angle, and the blue line shows the current of the motor load. The MCP joint ROM is $0-60^{\circ}$ and the flexion angular velocity of the finger is $60 / 0.58^{\circ} / \mathrm{s}$; (b) Measured grip force $(\mathrm{kg})$ of the developed myoelectric hand prosthesis. The black line shows the current of the motor load, and the red line shows the measured grip force.

The grip force of the hand is as important as the flexion speed of the finger. The developed hand was driven using a battery with a capacity of $2000 \mathrm{mAh}$. To reduce the battery consumption when the hand was driven, the grasping section of the hand was divided into a normal grasping section and a power grasping section. A PWM signal that sequentially increases the duty ratio from $0 \%$ to $90 \%$ was applied to the normal grasping section, and a PWM signal with a 90\% duty ratio was used in the power grasping section to 
apply a high current to the motor. An instantaneous high current was required in the power grasping section. When such a current was applied using the anti-reverse characteristic of the power transmission worm gear, the worm gear crossed the tooth surface of the worm wheel; the gears did not loosen, and the grasping power of the prosthetic hand gradually increased. Therefore, the measured grip force of the normal grasping section was $40 \mathrm{~N}$ or more, and that of the power grasping section was $120 \mathrm{~N}$ or more (see Figure $4 \mathrm{~b}$ ).

\subsection{Motion Classification Method}

Conventional myoelectric prosthetic hands are typically controlled by EMG signals from the residual muscles of upper limb amputees. However, because residual muscles generate very few EMG signals, upper limb amputees cannot perform various hand gestures and grip motions with these hands. By contrast, the developed hand can perform eight hand gestures and grip motions using only two EMG signals. Four motion states are used to perform these eight hand gestures and grip motions: a grip motion state and a gesture motion state classified by the EMG signal, and an adduction state and an abduction state respectively classified into the adduction/abduction motions of the thumb. To achieve this, as shown in Table 2, two EMG signals are divided into three control signals: a co-contraction signal (CC) that is used to classify the grip motion state and the gesture motion state of the prosthetic hand, and an open signal (OS) and an EMG close signal (CS) that are used to classify the eight hand gestures and grip motions.

Table 2. EMG control signal classification.

\begin{tabular}{cc}
\hline Command & Type of Function \\
\hline Co-Contraction (CC) & Mode change \\
\hline Open Signal (OS) & Motion (thumb extension state) \\
\hline Close Signal (CS) & Motion (thumb flexion state) \\
\hline
\end{tabular}

The most important hand function for upper limb amputees is the grip motion. Therefore, the normal motion state of the developed hand is the grip motion state. To perform hand gestures in the grip motion state, the developed hand should permit EMG CC signals. Additionally, to perform four grip motions in the basic grip motion state, the thumb must be classified into the adduction and the abduction states. These states are classified based on the value of the Hall Sensor (HS) mounted inside the palm of the developed hand. As shown in Figure 5, the thumb rotation angle can be classified into the adduction state and the $90^{\circ}$ abduction state.
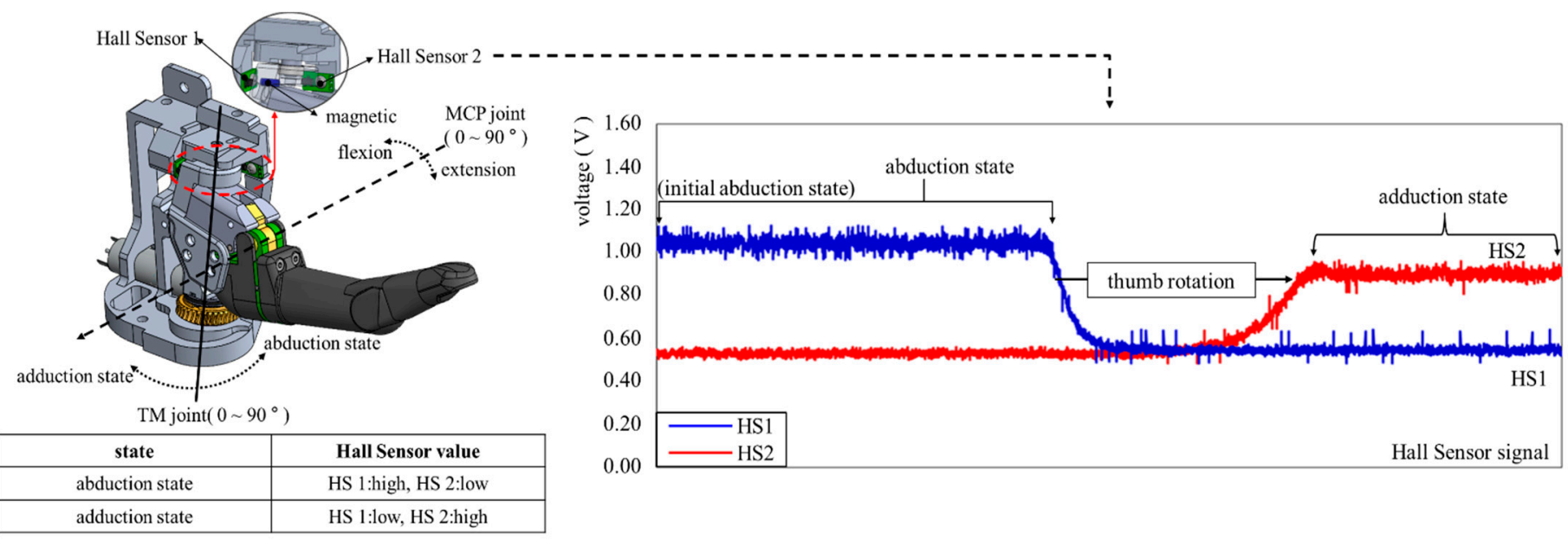

Figure 5. Adduction and abduction motion states of the thumb. The blue line shows the HS 1 value, and the red line shows the HS2 value. 


\subsection{Easy and Effective Control Algorithm}

We developed a practical control algorithm to enable upper limb amputees to use the developed hand easily and effectively. This algorithm uses the output of the CC, OS, and CS signals from the two EMG sensors to make the hand perform four hand gesture motions and four grip motions. Figure 6a shows the state transition diagram of the developed algorithm. The hand has a grip motion and gesture motion state, and the normal motion state is a grip motion. When the CC signal is inputted in the grip motion state, the hand switches to the gesture motion state, and when the CC signal is inputted again, the hand switches back to the grip motion state. When the grip motion state or gesture motion state is determined by the CC signal, the adduction or abduction state of the thumb is determined by the value of the HS mounted in the palm of the hand. If HS1 is high and HS2 is low in the grip motion state, an abduction state is applied. At this time, when the OS signal is applied, a hook grip with the thumb extension state is performed; when the CS signal is applied, a lateral grip with the thumb flexion state is performed. In the grip motion state, when HS1 is low and HS2 is high, an abduction state is applied. At this time, when the OS signal is applied, a power grip with the thumb extension state is performed, and when the CS signal is applied, a precision grip with the thumb flexion state is performed. When the CC signal is applied in the grip motion state (i.e., normal state), the hand changes to the gesture motion, and when HS1 is high and HS2 is low, an abduction state is applied. At this time, when the OS signal is applied, the thumb up gesture with the thumb extension state is performed, and when the CS signal is applied, the direction gesture with the thumb flexion state is performed. In the gesture motion state, if HS1 is low and HS2 is high, an adduction state is applied. At this time, when the OS signal is applied, the OK gesture with the thumb extension state is performed, and when the CS signal is applied, the victory gesture with the thumb flexion state is performed.

For example, when an upper limb amputee wants to perform a victory motion, in the grip motion state, the CC signal is applied to switch to the gesture motion state; the thumb is put into the adduction state, and the CS signal is applied to perform the victory gesture. Further, when an upper limb amputee wants to perform a lateral grip motion in the victory motion, the CC signal is applied to switch to the grip motions state; the thumb is put into the abduction state, and the OS signal is applied to perform the hook grip motion (Figure 6b). Thus, the upper limb amputees using the easy and effective control algorithm can perform various hand motions with two (CC, CS or CC, OS) control commands.

\subsection{Multifunctional Myoelectric Hand Prosthesis System}

We developed a multi-DOF myoelectric hand, a controller that can control each finger independently, and an easy and efficient control algorithm that allows for easy and efficient use of the hand. Figure 7 shows the multifunctional myoelectric hand prosthesis system for upper limb amputees. The hand has five fingers, each of which can be actuated by the controller with a six-axis motor driver to perform flexion and extension motions. For the thumb, adduction and abduction motions can be performed manually or automatically by driving the motor at the TM joint. Two EMG signals are used to perform the various hand gestures and grip motions of the developed hand. The EMG sensor used is a surface-dry type and has similar performance to the general EMG sensor used in Ottobock or Ossur [29]. The specifications of the EMG sensor are shown in Table 3; an electrical signal is output in an envelope type according to the movement of the muscle.

A lithium-ion battery with an input voltage of $7.4 \mathrm{~V}$ and a capacity of $2000 \mathrm{mAh}$ is used to drive the hand.

The human hand can perform various grip motions [30]. The developed system can perform four hand gestures-thumb up, OK, victory, and point-and four grip motionspower grip, hook grip, lateral grip, and tip grip-using the easy and effective control algorithm (see Figure 8). 


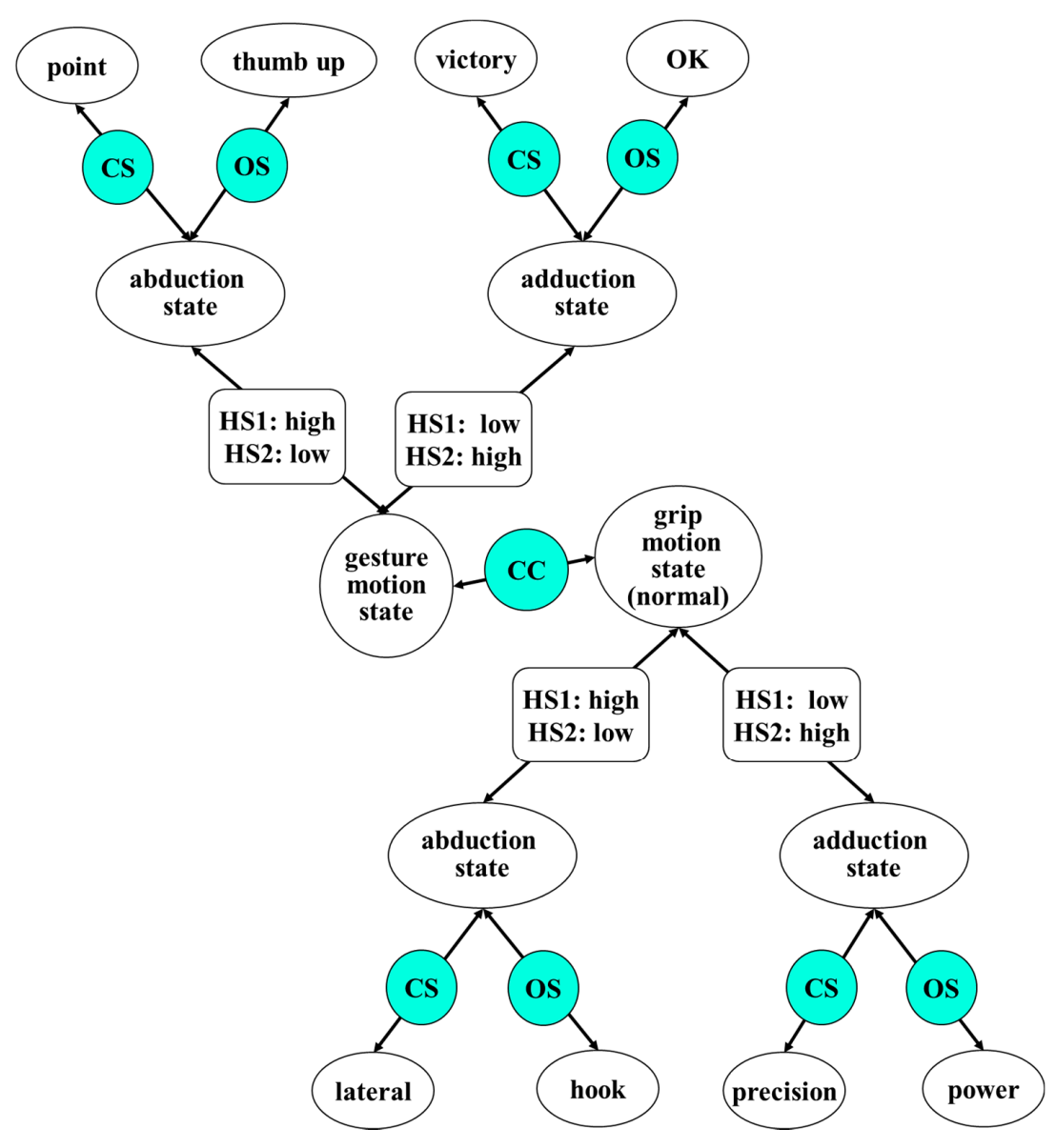

(a)

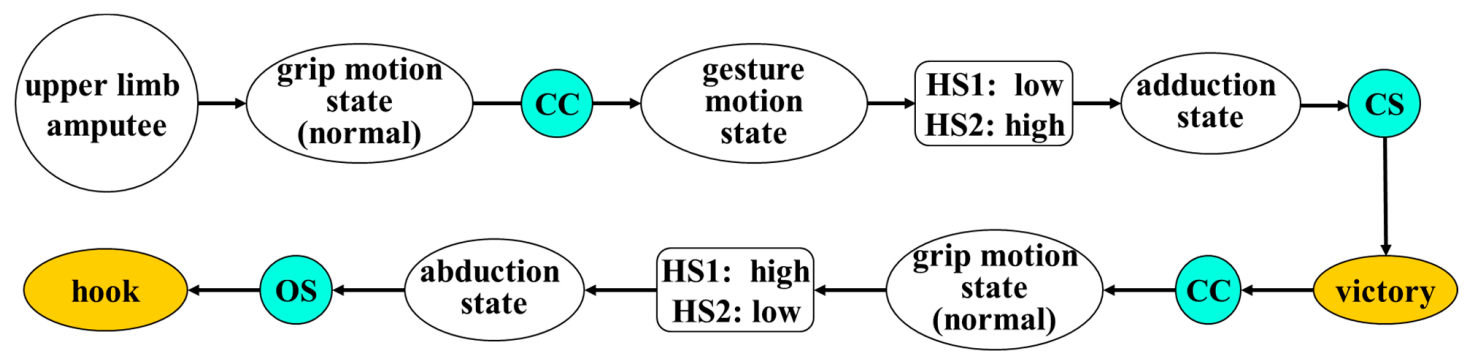

(b)

Figure 6. Developed control algorithm: (a) state transition diagram of the control algorithm and (b) state-transition sequence of the algorithm for performing a lateral grip motion in a victory hand gesture.

Table 3. Specifications of the EMG sensor.

\begin{tabular}{cc}
\hline & EMG Sensor [29] \\
\hline Gain & $2000-100,000$ \\
\hline Bandwidth & $90-330 \mathrm{~Hz}$ \\
\hline Rejection frequency & $60 \mathrm{~Hz}$ \\
\hline CMRR & Above $100 \mathrm{~dB}(1100 \mathrm{~dB})$ \\
\hline Noise & $47 \mu \mathrm{V} / \sqrt{ } \mathrm{Hz} @ 100 \mathrm{~Hz}$ \\
\hline Electrode & $3-$ points \\
\hline Phase margin & 75 \\
\hline
\end{tabular}




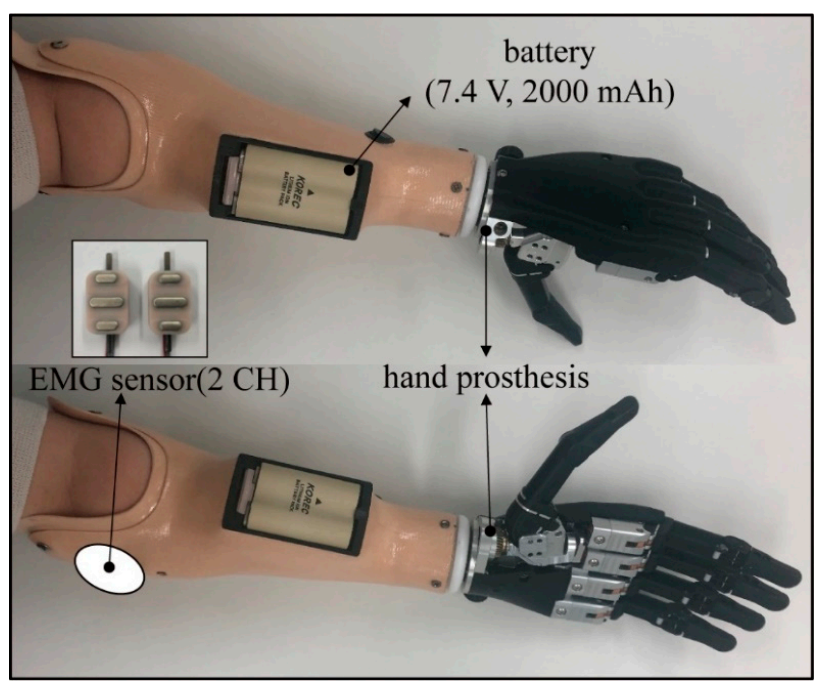

Figure 7. The developed multifunctional myoelectric hand prosthesis system.

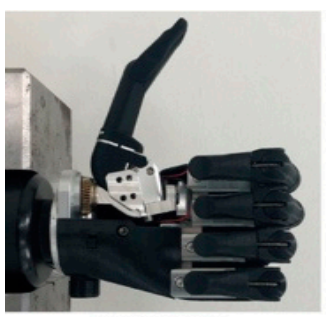

thumb up

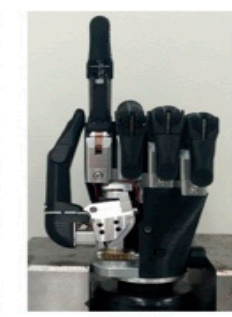

point

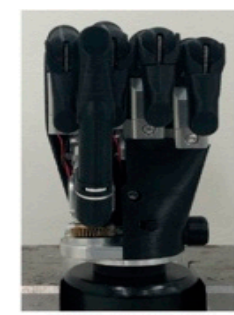

power grip

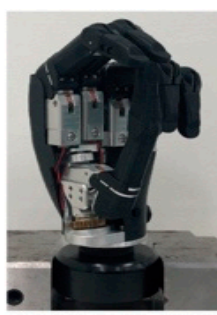

precision grip

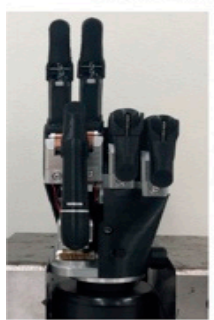

V

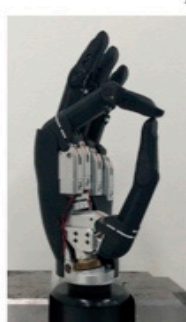

$\mathrm{OK}$

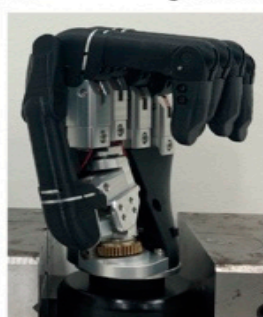

lateral grip

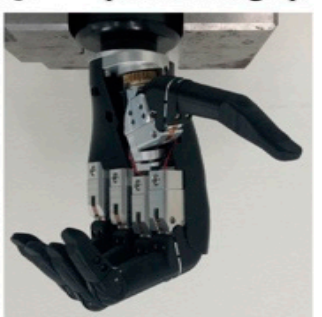

hook grip

Figure 8. Eight hand gestures and grip motions performable by the hand.

\section{Experimental Results}

For the clinical trial, Institutional Review Board approval and informed consent were obtained before beginning any of the experiments (IRB No. RERI-IRB-191230). The hand can perform four hand gestures used as a means of communication as well as four grip motions used commonly in the activities of daily life. Figure 9 shows the experimental results verifying that the hand can perform four hand gesture motions using the control algorithm.

We confirmed the performance of the algorithm developed for normal subjects in an earlier study [31]. The results indicated that a normal subject could easily and effectively perform various hand motions using the multi-DOF myoelectric hand prosthesis with only two EMG sensors. Thus, the EMG signals of an upper limb amputee were used in this experiment. The subject who participated in the experiment is a bilateral upper limb amputee who has been without the upper limbs for over 15 years. The residual amputation site is $80 \mathrm{~mm}$ above the elbow. The subject currently uses a three-finger type myoelectric hand prosthesis with one DOF, and the number of EMG signals generated from the residual muscles is two. In this study, two EMG sensors were installed on the extensor carpi radialis on the lateral side and on the flexor carpi ulnaris on the medial side. 


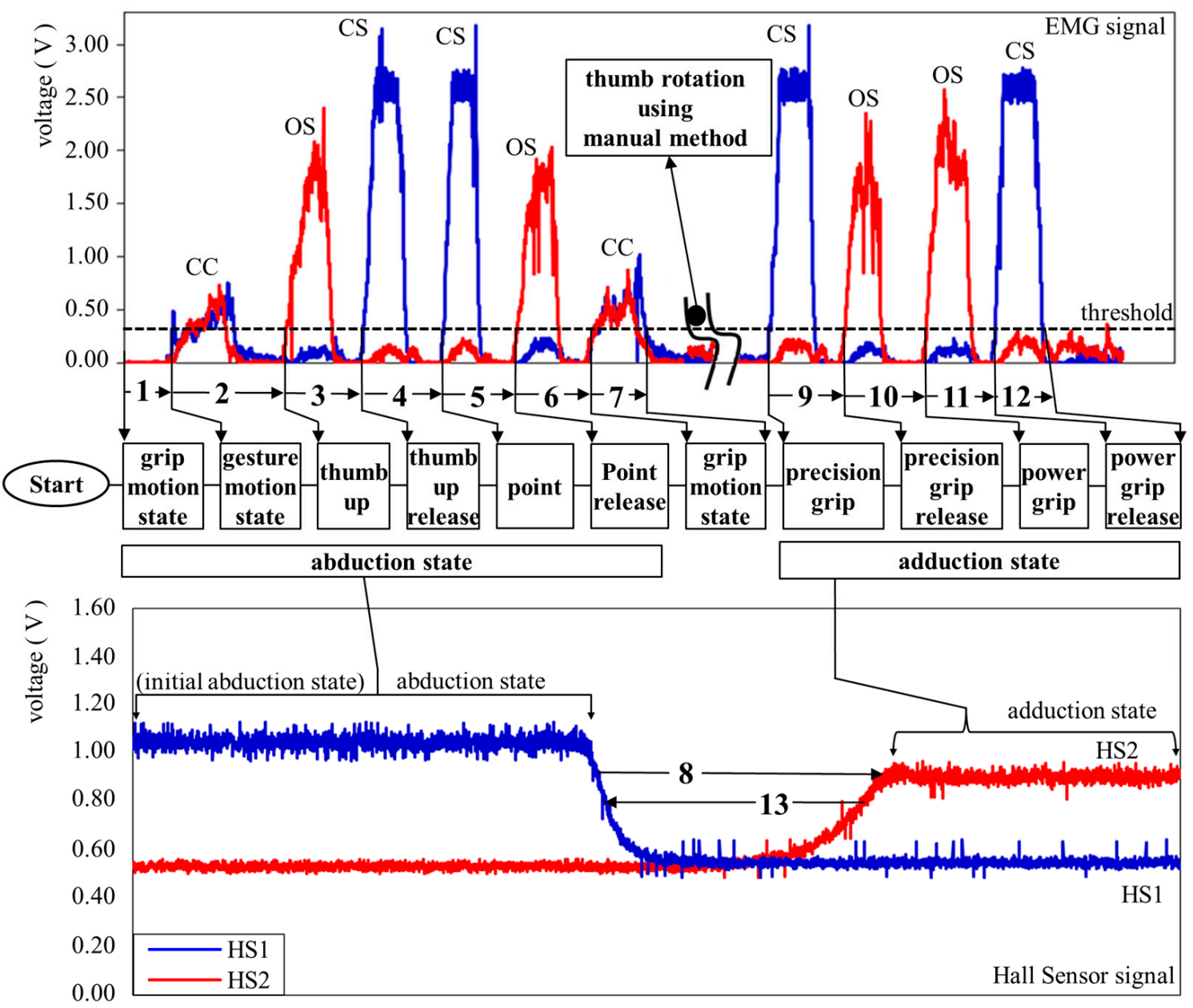

(a)

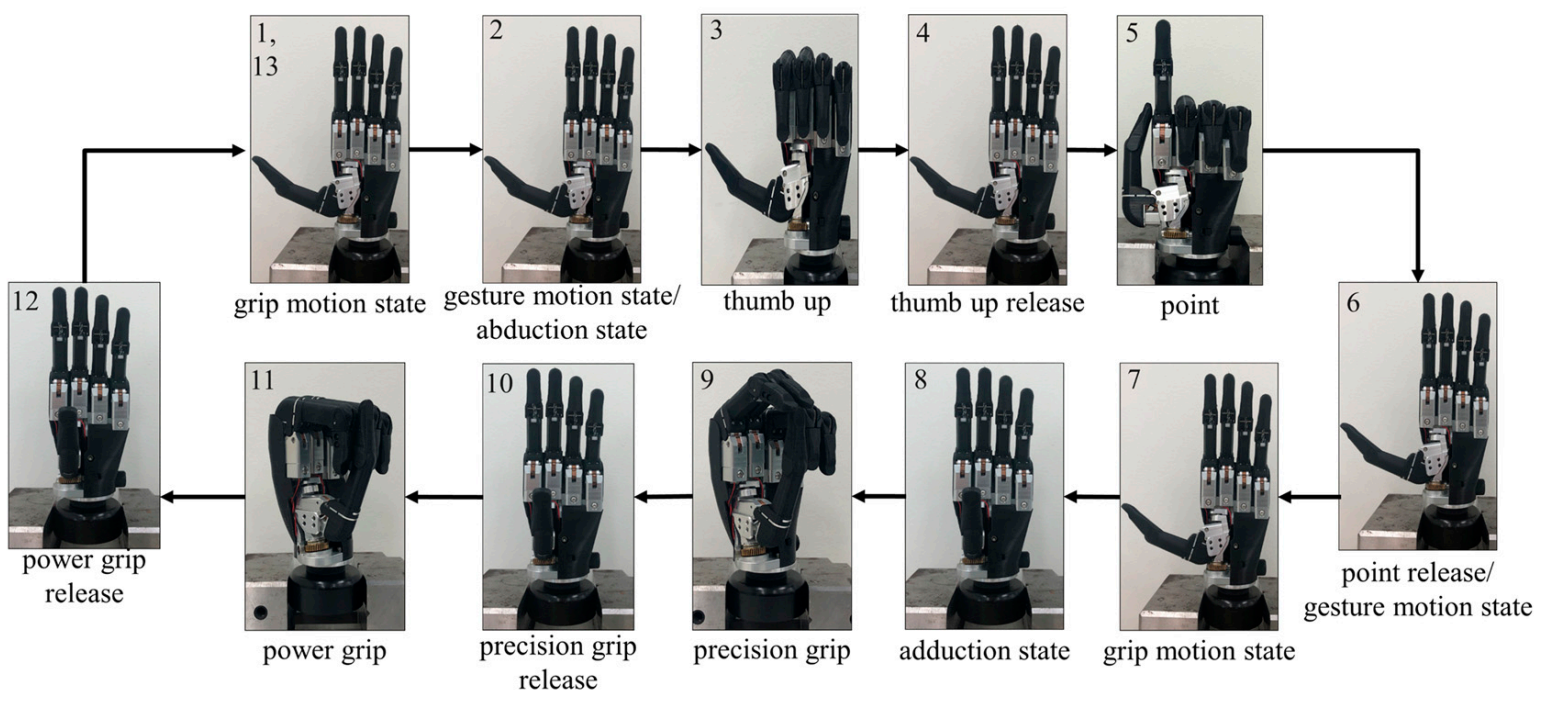

(b)

Figure 9. Experimental results obtained using the developed easy and efficient control algorithm: (a) EMG signals of an upper limb amputee and the hall sensor value according thumb position; (b) Results of the various hand motions performed using developed hand. 
Figure 9(1-12) shows that upon receiving an EMG CC signal, the hand changed from the grip motion state to the gesture motion state. At this time, because HS1 was higher than HS2, the hand was in the abduction state. In this state, upon applying an OS signal, the hand performed the thumb up gesture (see Figure 9(3)), and upon applying a CS signal, the hand performed the point gesture (see Figure 9(5)). Upon applying a CC signal, the hand returned to the gesture motion state and changed to the grip motion state (see Figure 9(6)). At this time, when the thumb was rotated manually to make HS2 higher than HS1, the hand entered the adduction state (see Figure 9(8)). In this state, upon applying a CS signal, the hand performed a precision grip motion (see Figure 9(9)), and upon applying an OS signal, the hand performed a power grip motion (see Figure 9(11)). These experimental results demonstrate that the developed hand can perform various hand gesture motions and grip motions through the control algorithm while using only two EMG signals.

\section{Conclusions}

Multi-DOF myoelectric hand prostheses are assistive devices for upper limb amputees to compensate for lost hand functions for upper limb amputees who lose their hand functions and complain of experiencing limitations in their daily living activities. However, as most upper limb amputees have less than two EMG signals generated at the amputation site, it is difficult to perform various hand gestures and grip motions using a multi-DOF myoelectric hand prosthesis. To overcome this problem, this paper proposed a multifunctional myoelectric hand prosthesis system that performs various hand motions using only two EMG sensors. The proposed system consists of a multi-DOF myoelectric prosthetic hand with five fingers, a controller, and an easy and effective control algorithm. The hand performs various hand motions using six DOFs, and its total weight is $503 \mathrm{~g}$. The proposed algorithm can control the eight hand motions according to the thumb adduction and abduction motion state and the thumb flexion and extension state using three control commands generated solely from the two EMG sensors. The results of experiments conducted on an upper limb amputee to validate the control algorithm confirmed that the developed system allows the easy performing of four hand gestures and four grip motions solely using the two EMG sensors.

In future work, the authors continue to evaluate the efficiency of the proposed algorithm by comparing the developed hand with commercially available multi-DOF myoelectric prosthetic hands, quantitatively. Further, we will conduct experiments with more upper limb amputees using the developed system.

Author Contributions: Conceptualization, S.-Y.J. and S.-H.P.; methodology, S.-Y.J. and S.-H.P.; software, S.-G.K.; validation, S.-Y.J. and S.-G.K.; investigation, S.-Y.J.; data curation, S.-Y.J. and S.-G.K.; writing—original draft preparation, S.-Y.J.; writing—review and editing, J.-H.K. and S.-H.P.; visualization, S.-Y.J.; supervision, S.-H.P.; project administration, S.-H.P. All authors have read and agreed to the published version of the manuscript.

Funding: This work was carried out with the support of "Cooperative Research Program for Agriculture Science and Technology Development (Project No. PJ015312012021)" Rural Development Administration, Republic of Korea.

Institutional Review Board Statement: The study was conducted according to the guidelines of the Declaration of Helsinki and approved by the Ethics Committee of Rehabilitation Engineering Research Institute (protocol code RERI-IRB-191230, 30 December 2019).

Informed Consent Statement: Informed consent was obtained from all subjects involved in the study.

Conflicts of Interest: The authors declare no conflict of interest. 


\section{References}

1. Antfolk, C.; D'Alonzo, M.; Controzzi, M.; Lundborg, G.; Rosen, B.; Sebelius, F.; Cipriani, C. Artificial redirection of sensation from prosthetic fingers to the phantom hand map on transradial amputees: Vibrotactile versus mechanotactile sensory feedback. IEEE Trans. Neural. Syst. Rehabil. Eng. 2012, 21, 112-120. [CrossRef] [PubMed]

2. Ottobock. Available online: https://www.ottobockus.com/prosthetics/upper-limb-prosthetics/solution-overview/myoelectricprosthetics / (accessed on 1 June 2021).

3. Belter, J.T.; Segil, J.L.; Dollar, A.M.; Weir, R.F. Mechanical design and performance specifications of anthropomorphic prosthetic hands: A review. J. Rehabil. Res. Dev. 2013, 50, 599-618. [CrossRef]

4. Bennett, D.A.; Dalley, S.A.; Truex, D.; Goldfarb, M. A multigrasp hand prosthesis for providing precision and conformal grasps. IEEE/ASME Trans. Mechatron. 2014, 20, 1697-1704. [CrossRef] [PubMed]

5. Ryu, W.; Choi, Y.; Choi, Y.J.; Lee, Y.G.; Lee, S. Development of an anthropomorphic prosthetic hand with underactuated mechanism. Appl. Sci. 2020, 10, 4384. [CrossRef]

6. Controzzi, M.; Clemente, F.; Barone, D.; Ghionzoli, A.; Cipriani, C. The SSSA-MyHand: A dexterous lightweight myoelectric hand prosthesis. IEEE Trans. Neural. Syst. Rehabil. Eng. 2016, 25, 459-468. [CrossRef] [PubMed]

7. Yong, X.; Jing, X.; Wu, X.; Jiang, Y.; Yokoi, H. Design and implementation of arch function for adaptive multi-finger prosthetic hand. Sensors 2019, 19, 3539. [CrossRef]

8. Dorcas, D.; Scott, R.N. A three state myoelectric control. Med. Biol. Eng. 1966, 4, 367-370. [CrossRef]

9. Dorcas, D.; Dunfield, V.A.; Scott, R.N. Improved myoelectric control systems. Med. Biol. Eng. 1970, 8, 333-341. [CrossRef]

10. Hudgins, B.; Parker, P.; Scott, R.N. A new strategy for multifunction myoelectric control. IEEE Trans. Biomed. Eng. 1993, 1, 82-94. [CrossRef] [PubMed]

11. Englehart, K.; Hudgin, B.; Parker, P.A. A wavelet-based continuous classification scheme for multifunction myoelectric control. IEEE Trans. Biomed. Eng. 2001, 48, 302-311. [CrossRef]

12. Chu, J.U.; Moon, I.; Lee, Y.J.; Kim, S.K.; Mun, M.S. A supervised feature projection-based real-time EMG pattern recognition for multifunction myoelectric hand control. IEEE/ASME Trans. Mechatron. 2007, 12, 282-290. [CrossRef]

13. Scheme, E.; Fougner, A.; Stavdahl, Ø.; Chan, A.D.; Englehart, K. Examining the adverse effects of limb position on pattern recognition based myoelectric control. In Proceedings of the 2010 Annual International Conference of the IEEE Engineering in Medicine and Biology (EMBC 2010), Buenos Aires, Argentina, 31 August-4 September 2010; pp. 6337-6340.

14. Cipriani, C.; Controzzi, M.; Kanitz, G.; Sassu, R. The effects of weight and inertia of the prosthesis on the sensitivity of electromyographic pattern recognition in relax state. JPO J. Prosthet. Orthot. 2012, 24, 86-92. [CrossRef]

15. Atzori, M.; Hager, A.-G.M.; Elsig, S.; Giatsidis, G.; Bassetto, F.; Müller, H. Effect of clinical parameters on the control of myoelectric robotic prosthetic hands. J. Rehabil. Res. Dev. 2016, 53, 345-358. [CrossRef] [PubMed]

16. Naik, G.R.; Al-Timemy, A.H.; Nguyen, H.T. Transradial amputee gesture classification using an optimal number of sEMG sensors: An approach using ICA clustering. IEEE Trans. Neural. Syst. Rehabil. Eng. 2016, 24, 837-846. [CrossRef] [PubMed]

17. Vujaklija, I.; Farina, D.; Aszmann, O.C. New developments in prosthetic arm systems. Orthop. Res. Rev. 2016, 8, 31-39. [CrossRef] [PubMed]

18. Jiralerspong, T.; Nakanishi, E.; Liu, C.; Ishikawa, J. Experimental study of real-time classification of 17 voluntary movements for multi-degree myoelectric prosthetic hand. Appl. Sci. 2017, 7, 1163. [CrossRef]

19. Pancholi, S.; Joshi, A.M. Electromyography-based hand gesture recognition system for upper limb amputees. IEEE Sens. Lett. 2019, 3, 1-4. [CrossRef]

20. Jafarzadeh, M.; Hussey, D.C.; Tadesse, Y. Deep learning approach to control of prosthetic hands with electromyography signals. In Proceedings of the 2019 IEEE International Symposium on Measurement and Control in Robotics (ISMCR), Houston, TX, USA, 19-21 September 2019; p. A1-4.

21. Tam, S.; Boukadoum, M.; Campeau-Lecours, A.; Gosselin, B. Intuitive real-time control strategy for high-density myoelectric hand prosthesis using deep and transfer learning. Sci. Rep. 2021, 11, 1-14. [CrossRef]

22. Iqbal, N.V.; Subramaniam, K. A review on upper-limb myoelectric prosthetic control. IETE J. Res. 2018, 64, 740-752. [CrossRef]

23. Parker, A. Control of upper limb prostheses: Terminology and proportional myoelectric control-A review. IEEE Trans. Neural. Syst. Rehabil. Eng. 2012, 20, 663-677.

24. Graupe, D.; Cline, W.K. Functional separation of EMG signals via ARMA identification methods for prosthesis control purposes. IEEE Trans. Syst. Man. Cybern. 1975, SMC-5, 252-259. [CrossRef]

25. Doerschuk, P.C.; Gustafon, D.E.; Willsky, A.S. Upper extremity limb function discrimination using EMG signal analysis. IEEE Trans. Biomed. Eng. 1983, BME-30, 18-29. [CrossRef] [PubMed]

26. Li, G. Electromyography pattern-recognition-based control of powered multifunctional upper-limb prostheses. In Advances in Applied Electromyography; InTech: Rijeka, Croatia, 2011.

27. Ossur. Available online: https://www.ossur.com/en-us/prosthetics/touch-solutions (accessed on 1 June 2021).

28. Carrozza, M.C.; Cappiello, G.; Micera, S.; Edin, B.B.; Beccai, L.; Cipriani, C. Design of a cybernetic hand for perception and action. Biol. Cybern. 2006, 95, 629-644. [CrossRef]

29. Park, S.H.; Hong, B.K.; Kim, J.K.; Hong, E.P.; Mun, M.S. Development of the myoelectric hand with a 2 DOF auto wrist module. J. Inst. Control. Robot. Syst. 2011, 17, 824-832. [CrossRef] 
30. Feix, T.; Pawlik, R.; Schmiedmayer, H.-B.; Romero, J.; Kragic, D. A comprehensive grasp taxonomy. In Proceedings of the Robotics, Science and Systems: Workshop on Understanding the Human Hand for Advancing Robotic Manipulation, Seattle, WA, USA, 28 June-1 July 2009; Volume 2, pp. 2-3.

31. Jung, S.Y.; Kim, S.G.; Jang, D.; Kim, S.K.; Park, S.H.; Kim, J.H. Development of the multi-DOF myoelectric hand prosthesis with the intuitive control algorithm. J. Korean Soc. Precis. Eng. 2020, 37, 139-147. [CrossRef] 